\title{
MEJORAMIENTO DE LOS FALSOS RECHAZOS GENERADOS POR LOS INSPECTORES DE BOTELLA VACÍA POR MEDIO DE UN CONTROL ESTADÍSTICO DE PROCESOS (CEP)
}

\section{IMPROVEMENT OF THE FALSE REJECTIONS GENERATED BY THE INSPECTORS OF EMPTY BOTTLES BY MEANS OF A STATISTICAL PROCESS CONTROL (SPC)}

\author{
Martha Catalina Ospina Hernández * , Albert Miyer Suarez Castrillon ** , Elkin \\ Gregorio Flórez Serrano**, José Roberto Marín* \\ * Universidad Nacional Abierta y a Distancia (UNAD), Facultad de Ingenierías, \\ Programa de Ingeniería Industrial. \\ Dirección, Medellín, Antioquia, Colombia. \\ Teléfono y Fax, con indicativos internacional y nacional. \\ E-mail: \{martha.ospina, ajmonto7029\}@unad.edu.co. \\ *** Universidad de Pamplona, Facultad de Ingenieria y Arquitectura, Grupo GIMUP. KM \\ 1 via Bucaramanga, Pamplona, Norte de Santander, Colombia. \\ 00575685303 \\ E-mail: \{albertmiyer, eflorez\}@ unipamplona.edu.co.
}

\begin{abstract}
Resumen: En el siguiente trabajo se desarrolla una propuesta para mejorar e implementar el plan de manejo actual de los inspectores de botella vacía en una cervecería de la ciudad de Medellín, identificando los puntos que afectan la productividad, y cómo se puede implementar un mantenimiento y manejo estandarizado de maquinaria centrado en confiabilidad, en este caso específicamente los inspectores de botella vacía. Para el desarrollo del trabajo, se utiliza el Control Estadístico de Procesos (CEP), además se analizan alternativas y se analiza el tipo de mantenimiento y manejo que es factible de implementarse en la empresa, tambien se realiza estudio sobre el funcionamiento de algunas líneas de producción que presenta mayor número de falsos rechazos de envase, en todas las áreas que afectan directa e indirectamente las líneas, las cuales son: El talento humano, el manejo de repuestos, el presupuesto, los procedimientos y la programación de mantenimiento determinando falencias y fortalezas del plan de mantenimiento utilizado actualmente.
\end{abstract}

Palabras clave: Normativa de publicación, procedimientos, publicación, formato IFAC. 
Abstract: In the following work a proposal is developed to improve and implement the current management plan of the empty bottle inspectors in a brewery in the city of Medellín, identifying the points that affect productivity, and how standardized maintenance and management can be implemented of machinery focused on reliability, in this case specifically the empty bottle inspectors. For the development of the work, Statistical Process Control (CEP) is used, alternatives are analyzed and the type of maintenance and management that is feasible to be implemented in the company is analyzed, as well as a study on the operation of some lines of work. production that presents the highest number of false rejects of packaging, in all areas that directly and indirectly affect the lines, which are: Human talent, spare parts management, budget, procedures and maintenance schedule determining flaws and strengths of the maintenance plan currently used.

Keywords: empty container inspectors, SPC, failures.

\section{INTRODUCCIÓN}

Uno de los aspectos importantes en toda organización para la optimización de los procesos productivos, es el empleo de herramientas computacionales para la estandarización de un proceso en beneficio de la reducción de tiempos, insumos, falsos rechazos, espacio y tiempos de almacenaje, entre otros, el cual lleva a resultados positivos en la cadena productiva, mejorando con ello los procesos manufactureros y la optimización de recursos dentro de la organización (Ospina, et al. 2017).

El mantenimiento de maquinaria industrial ha alcanzado un valor muy importante dado que la competencia se está desarrollando rápidamente en cuanto al uso de medios tecnológicos se refiere. Se llevan a cabo muchos esfuerzos para lograr los objetivos de incremento de la disponibilidad de los equipos y disminución de la tasa de fallos, objetivos que se deben obtener teniendo en cuenta el costo - beneficio del mantenimiento.

El mantenimiento tiene uno de los costos de operación más altos que deben ser vigilados en la industria, pero se debe ser consecuente al momento de evaluar el impacto comercial, volumen de producción, calidad de producción, costos operacionales, seguridad y riesgo ambiental tomando como referentes las bases de datos generadas de la empresa, se realiza un control estadístico de procesos (CEP) para medir y disminuir la cantidad de fallos presentados por los inspectores de botella vacía; este control permitirá hacer un análisis adecuado y organizado para poder ejecutar un estudio de eficiencias y afectaciones de los equipos en producción y sugerir el seguimiento del diagrama de toma de decisiones de los manuales de cada equipo. Dentro de este se enfoca el estudio de datos a los equipos más críticos, identificados como los inspectores de envase vacío que presentan el mayor número de eventos de falla y tiempos perdidos de producción, para mejorar la eficiencia mecánica de los mismos, esto a través de prácticas de producción estandarizadas.

\section{ANTECEDENTES}

La embotelladora en donde se llevó a cabo el estudio, como empresa organizada tiene sus propios parámetros de trabajo y de manejo de eficiencias en las líneas de producción, esto con el fin de mantener la inversión realizada, aumentar las ganancias y llenar las expectativas de los clientes.

De acuerdo con (García et al., 2003; Campos,2009) es importante establecer un sistema de gestión para la calidad que este claramente orientado a los procesos y a la mejora continua; Pues, las organizaciones lograrán el liderazgo en la medida que tengan la habilidad para mantener la excelencia de sus procesos y se comprometan con el constante desarrollo de sus objetivos, siempre orientados a la satisfacción de sus clientes.

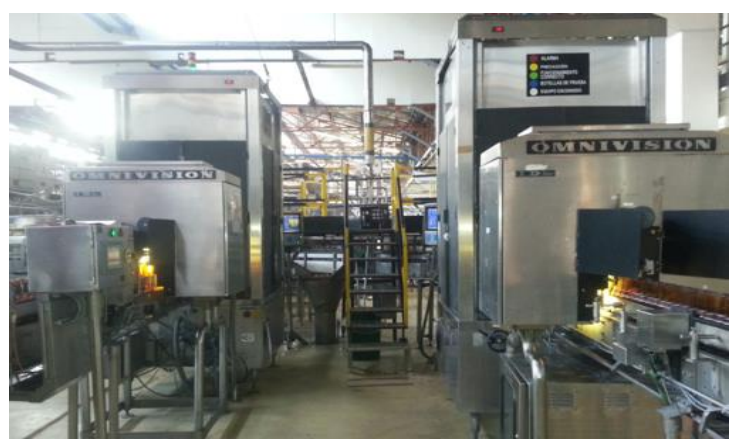

Fig. 1 Inspectores de botella

Esta empresa manufacturera, es una empresa que forma parte de una multinacional que ha llegado a diferentes partes del mundo, por lo tanto, debe cumplir un estándar de calidad y de producción, por ello todas sus líneas de producción deben operar de manera que sobrepasen o mantengan 
estables los parámetros de eficiencia productiva y mecánica.

\section{DIAGNÓSTICO INICIAL}

El presente trabajo inicia con la recolección de información por medio de CEP, esto para realizar ajustes en los inspectores de botella, esta información ha sido extraída de manuales de las empresas que fabrican y comercializan las máquinas, del mismo modo que de las que prestan soporte técnico a nivel mundial, nacional y local.

Gran parte de la información fue recopilada durante capacitaciones recibidas en diferentes partes y de diferentes entidades y relacionadas a la interacción con los inspectores de botella vacía.

La inspección que se realiza a una botella se basa en los siguientes parámetros: base, pared interna, labio, pared externa y líquido residual, estas son las zonas que normalmente se inspeccionan a una botella de vidrio

En la siguiente imagen (figura 2) se muestran los porcentajes de rechazos tan altos que se tenían antes de hacer las respectivas calibraciones y ajustes especialmente en la línea 750 .

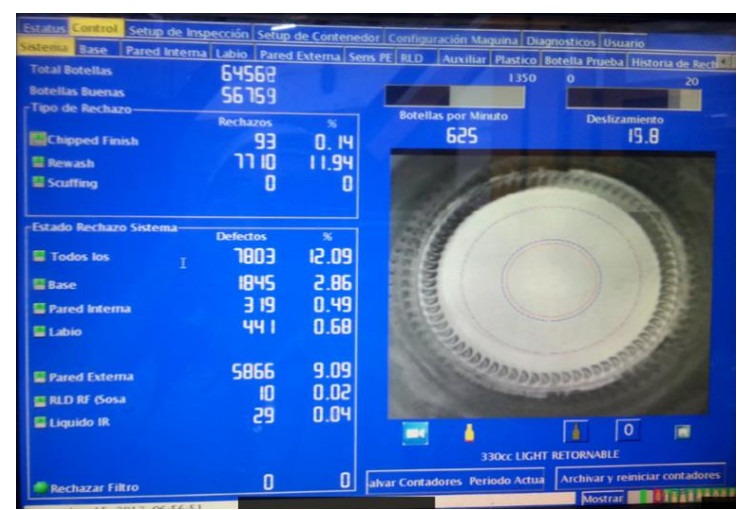

Fig. 2 Porcentajes de rechazo de la línea 750

Dicho lo anterior, para permanecer en el mercado, las empresas han tenido que desarrollar numerosos enfoques, y utilizar distintas herramientas, con el propósito de aumentar su productividad. Una de las herramientas que más comúnmente usan las empresas para lograr una alta productividad es la medición de sus procesos mediante indicadores de productividad y eficiencia. Es así como la intención de realizar una repotenciación de la desempacadora de botellas Remy está dirigida a mejorar dichos indicadores; esto dado que con ello se aumentará su productividad y eficiencia.

\section{Desarrollo teórico}

\section{A. Control estadístico del proceso-CEP}

Actualmente existen, una gran variedad de herramientas que pueden ser utilizadas para posibles mejorías y diagnósticos, pero una de las principales es el uso de técnicas estadísticas que viene a lo largo de los años, mejorando todo sistema operacional además de permitir tornar los productos fabricados más competitivos. Debido a eso, el investigar las causas de problemas y caminos para mejorías debe ser constante.

Dentro de esas herramientas, una muy utilizada es el Control Estadístico de Proceso (CEP) que es la rama de calidad que consiste en la recolección, análisis e interpretación de datos, establecimiento de calidades, comparación de desempeños, verificación de desvíos, todo eso para su utilización en las actividades de mejoría y control de calidad de productos, servicios y diagnóstico de defectos.

Además de obtener productos con mejor calidad, la utilización del CEP genera costos menores y eso disminuye principalmente en función de dos razones: la inspección por muestreo y la reducción de rechazo. Donde la selección de muestras es de tamaño mucho menor que la población, disminuyendo consecuentemente los costos y paradójicamente acaba representando mejor las características de la población, otra ventaja en la reducción de costos es que el número y porcentaje de piezas defectuosas producidas en la fábrica van a disminuir con las mejorías en la línea de producción (Campos,2009).

El CEP permite que las acciones correctivas sean aplicadas antes del surgimiento de inconformidades, responde a la pregunta si el proceso está funcionando como debía o si está fuera de las especificaciones de calidad y ejecuta acciones apropiadas para lograr y mantener un estado de control estadístico (Wheeler, D;2010)

La utilización de esa herramienta permite conocer el comportamiento del proceso y hacer previsiones sobre su desempeño, ese comportamiento es analizado a través de medidas a él asociadas teniendo en cuenta los conceptos de estabilidad y capacidad. Un proceso es considerado estable si el mismo es reproducible. La estabilidad permite prever el desempeño del proceso en ejecuciones futuras y elaboración de planes alcanzables. Según (Ribeiro, J;2003). la previsibilidad es la esencia del 
control estadístico. Por otro lado, un proceso solo puede ser llamado eficaz, si él es estable y cumple con todos los objetivos y metas de la empresa y del cliente. Con relación a la estabilidad, es importante destacar que todos los procesos sufren variaciones en su comportamiento. Siendo así, un proceso estable no es aquél que no presenta variaciones, sino que presenta variaciones aceptables dentro de límites previsibles, que caracterizan la repetitividad de su comportamiento (Campos, R;2003)

Es muy común en fábricas donde los procesos industriales no son optimizados en el sentido de ser caracterizados por altos niveles de eficiencia, sin embargo, dentro del CEP existen herramientas para monitorizar el proceso y, por tanto, mejorarlo. El monitoreo tiene como requisitos el muestreo hecho periódicamente y tamaño de la muestra adecuado.

La principal ventaja proporcionada por esa herramienta es la realimentación del proceso en tiempo real, debiendo ser continua y estadísticamente fundamentada. Para mejorar la eficiencia de un servicio es necesario conocer con la situación en cada momento y especialmente en el caso en que se desea iniciar un proceso de transformación profundo.

\section{B. Fallas}

En los departamentos de mantenimiento industrial hay que tener bien definido el concepto de una falla, donde se debe entender o definir como toda alteración o interrupción en el cumplimiento de la función requerida; esta definición nos ayudan a revisar cómo se pueden originar y como clasificar las fallas de los equipos de la compañía. El origen de las fallas se puede presentar desde un mal diseño o error de cálculo

\section{ANÁLISIS DE RESULTADOS}

\section{A. Plan de implementación}

Teniendo en cuenta la disponibilidad de la empresa y los resultados del CEP y los estudios realizados se determina el siguiente modelo para el plan de implementación:

La primera etapa se compone de la capacitación del personal por departamentos, la temática a trabajar es unificar parámetros de trabajo y de lenguaje, no solo en el departamento de mantenimiento sino en producción también, así mismo la planta genera un lenguaje y una codificación de máquinas y de fallas organizada, permitiendo que las propuestas que se realicen para optimizar tiempos y procedimientos, den mayor resultado.

Inicialmente se busca generar interés por esta iniciativa, vendría una parte de teoría que sería básicamente para conocer las herramientas necesarias y como se deben usar, para que todos aporten a la consecución de información real y de valor, de todos los aspectos que se pueden mejorar en el funcionamiento del equipo de mantenimiento de maquinaria. $\quad$ Esta primera etapa debe tener una intensidad horaria que se puede acomodar a la disposición de la empresa.

También dentro de las capacitaciones se debe hacer consiente a todo el personal de la importancia y la responsabilidad que tiene cada uno en el momento de manejar datos y generar o modificar ordenes de trabajo, de servicio, solicitud de materiales, requisición de materiales, proyectos de mejora, etc.

En la segunda etapa se mejorara el uso de la documentación que ya se está manejando en la empresa como lo son las hojas de inspección y el cumplimiento real de los recorridos de inspección.

En la tercera etapa se generara la rotulación de los puntos de lubricación no solo de los equipos críticos sino en todas las máquinas y/o equipos de la línea de producción con la información no solo del lubricante que se le adiciona sino también la frecuencia y la fecha en que se le realizo la última intervención, así se genera menos paradas por falta de lubricación y extiende la vida útil de los engranajes en la línea de producción.

En la cuarta etapa se empezarán a terminar los trabajos por completo en los fines de semana, porque cuando se realiza una intervención los fines de semana se realizan las pruebas de seguridad, de funcionamiento y ajustes. De esta forma se busca disminuir trabajos correctivos en las horas de producción, que generan pérdida de tiempo, bajando las eficiencias en el arranque de la semana.

Adicionalmente a las etapas propuestas en el plan de implementación se sugiere tener en cuenta para las intervenciones de las fallas registradas en las máquinas.

B. Ajuste de los equipos

C. Los equipos fueron ajustados y evaluados día a día, en donde se observa una disminución importante de los fallos. Se hacen cálculos. 
Este análisis se realiza en compañía del Ingeniero de planta

D. En el salón de envase son 3 líneas de producción, pero el cálculo se hace solo con dos líneas, dado que la línea 750 se está alternando con la línea 5 o la línea 8 , lo que quiere decir que durante todo el año hay dos líneas de vidrio produciendo simultáneamente.

Tabla 1. Datos ajustados al inspector de botellas vacías

\begin{tabular}{|l|r|}
\hline $\begin{array}{l}\text { Promedio Turno botellas rechazadas } \\
\text { (antes) }\end{array}$ & 27.216 \\
\hline $\begin{array}{l}\text { Promedio Turno botellas rechazadas } \\
\text { (Después) }\end{array}$ & 21.409 \\
\hline Diferencia & 5.807 \\
\hline Promedio día & 17.421 \\
\hline $\begin{array}{l}\text { Costo de lavado de una botella en } \\
\text { reproceso }\end{array}$ & 87.105 \\
\hline Costo mensual & 2.090 .520 \\
\hline Costo anual por línea & 25.086 .240 \\
\hline Costo para dos líneas & 50.172 .480 \\
\hline Incremento de producción, Horas & 0,241958333 \\
\hline Ahorros por eficiencia, día & 411.329 \\
\hline Ahorros por eficiencia, mes & 9.871 .900 \\
\hline Ahorros por eficiencia, Año & 118.462 .800 \\
\hline Ahorro Total por linea & 143.549 .040 \\
\hline Ahorro Total por dos lineas & 287.098 .080 \\
\hline
\end{tabular}

E. Se adjunta evidencia de los datos ajustados al inspector de botellas vacías de la línea 750 con el formato de Light, al igual que este formato se ajustaron para marrón 750 en este equipo, en la línea 5 se ajustaron los formatos de ámbar 225, costeñita, marrón 330, light 330, formato de Trophy y en la línea 8 marrón 330, light 330 .

F. Una vez realizados los ajustes pertinentes a los equipos mencionados, se han alcanzado valores muy significativos como se muestra en la imagen 3 , de $2,43 \%$, esto siempre y cuando la botella no presente novedades, en términos de falsos rechazos. (Botellas que son rechazadas sin causa aparente sino por ajustes propios del inspector).

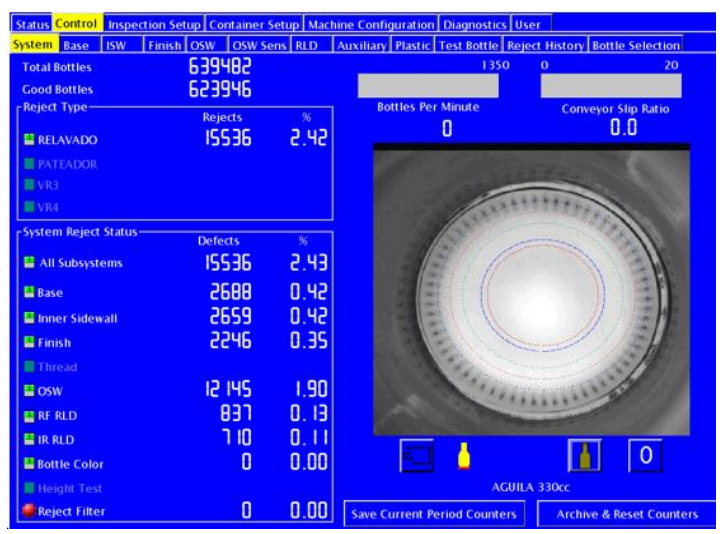

Fig. 3 Disminución de falsos rechazos

Para esta última etapa se ha socializado con los técnicos de línea la importancia de manejar unos buenos algoritmos de inspección, para esto se realizaron varias secciones de entrenamiento y capacitación como se muestra en las evidencias a continuación. Para soportar dichas capacitaciones se tiene el aval del ingeniero Diego Andrés Mejía que es el coordinador de mantenimiento de envase

Las capacitaciones fueron realizadas en distintos horarios dadas las circunstancias y los diferentes tiempos de disponibilidad de los técnicos, incluso desde los ajustes iniciales ya que fueron los de mayor beneficio dado que se pudieron apreciar los efectos.

Una vez realizadas las capacitaciones se han hecho acompañamientos a los técnicos para mejorar cada día el desempeño de los equipos, también se han dado tips en donde los técnicos identifican las posibles causas por las cuales no se logra una buena inspección, como por ejemplo una placa muerta mal ajustada, un Trigger mal alineado, los lentes sucios, una imagen mal definida y todo lo concerniente a una buena inspección.

\subsection{RESULTADOS OBTENIDOS}

Como se puede evidenciar en las imágenes se han logrado valores de rechazo muy significativos ya que se deja de reprocesar una gran cantidad de botellas que como se mostró y evidenció en el cálculo realizado tiene un valor muy alto cuantificándose en un año en dos líneas de envasado.

La línea 750 ha sido una de las líneas en donde mayores beneficios se han tenido puesto que con un rechazo del 12 o $14 \%$ es muy difícil lograr que la línea tenga continuidad y de una buena eficiencia, ésta en la actualidad maneja valores de 4 o $5 \%$ que aunque es alto es bueno puesto que esta 
botella por su tamaño y contorno presenta muchos defectos de fabricación.

Se hace el levantamiento de información valiosa que queda en un manual como fuente de consulta en el momento requerido e incluso en medios magnéticos. Se afianzan más los conocimientos de los técnicos para afrontar el día a día en la interacción con los equipos.

\section{CONCLUSIONES}

Se extrae información valiosa de los equipos y se compara con todos los inspectores para estandarizar el manejo de los equipos.

Se realizan las capacitaciones y se concientiza a los técnicos de la importancia de manejar estándares, se hace más fácil el trabajo cuando se hace en equipo y bajo los mismos principios.

Centralización del sitio de consulta como lo es el manual que se construyó, para canalizar dudas e inquietudes en los momentos requeridos.

Consolidación del trabajo en equipo puesto que con el transcurrir del tiempo se han aclarado dudas y se ha buscado alternativas que ayudan al equipo y a la empresa. Y mejora la productividad desde todo punto de vista, primero porque al tener menos rechazo las líneas tienen más continuidad y se generan menos procesos.

Con el análisis de modos y efectos de falla realizado a los equipos críticos de la línea y teniendo en cuenta el número de prioridad de riesgo (RPN), ejecutando las modificaciones del plan de mantenimiento propuesto y utilizando el plan de implementación con las etapas propuestas.

\section{RECOMENDACIONES}

- De acuerdo con los resultados obtenidos, se recomienda que para elevar la eficiencia promedio de la línea y mantenerla constante, se debe empezar por facilitar herramientas y capacitar mejor a las personas encargadas de registrar la base de datos de la operación de equipos como el Inspector de envase, para que sea posible identificar cual es realmente el evento que se presenta, y si es en la parte operativa o mecánica del equipo. El registro de la información de los tiempos perdidos debe ser claro y preciso. En repetidas ocasiones se encuentran incoherencias en tiempos perdidos registrados y no son claros los avisos generados de la parada y la causa de la falla.

\section{REFERENCIAS}

Ospina, M., Angulo, J. Suarez, A. y Suarez, S. (2017). Mejora del sistema de alimentación en las máquinas envasadoras multipack y rovema para reducir el riesgo ergonómico y los tiempos improductivos de operación en empresas manufactureras de chocolates. Revista Colombiana de Tecnologías de Avanzada. Vol. 1, Num. 29

Campos, V; (1992) TQC.: Controle da Qualidade Total (estilo japonês). Belo Horizonte: Fundação Christiano Ottoni, Escola de Engenharia da UFMG,

C. J. Torres, J. F. Archila, M. L. Tronco, M. Becker, A. J. V. Porto, A. J. Tiberti. (2013). Estudio cinemático de una plataforma robótica para agricultura. REVISTA COLOMBIANA DE TECNOLOGÍAS DE AVANZADA, ISSN: 1692-7257. 2(22).

Torres, C., Archila, J., Tronco, M., Becker, M., Viera Porto, A., \& Tiberti, A. (2013). Estudio cinemático de una plataforma robótica para agricultura. REVISTA COLOMBIANA DE TECNOLOGÍAS DE AVANZADA, 2(22).

Wheeler, D. J.; Chambers, D. S. (2010)Understanding Statistical Process Control. 3a ed. SPC Press, Knoxville Tennessee,

Ribeiro, J. ; C. S. Caten, (2012) Série Monográfica Qualidade - Controle Estatístico do Processo. Porto Alegre: FEENG/UFRGS, García, M. Quispe, C. and Ráez, L., (2003). "Mejora continua de la calidad en los procesos, " Industrial Data, Vol. 6, no. 1, pp. 89-94

Montgomery, D.C., (2009). Introduction to Statistical Quality Control 6th ed., John Wiley $\&$ Sons.

Minitab (2000). Minitab (Version 13.0) [Computer software]. State College, PA: Author.

Campos, R.; Rocha, R. (2009) "O controle estatístico de processos (CEP) para o monitoramento da qualidade do farelo lex no processo do óleo de soja na empresa CAC". n: IV Encontro de Produção Científica e Tecnológica, 4, 2009, Campo Mourão - PR. Anuais. Campo Mourão: NUPEM, , p. 13. 